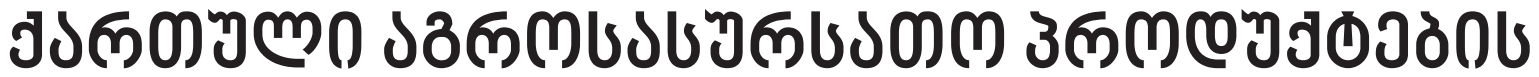

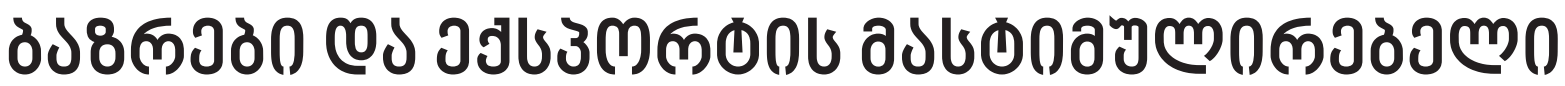

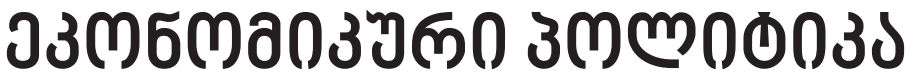

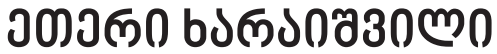

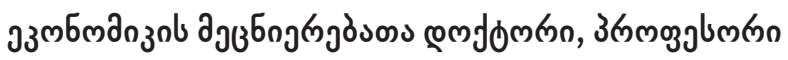

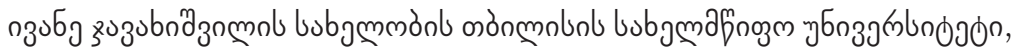

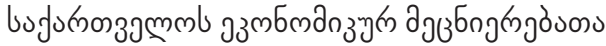

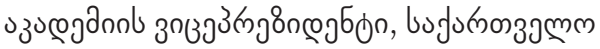

eter.kharaishvili@tsu.ge

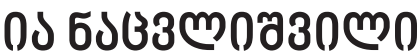

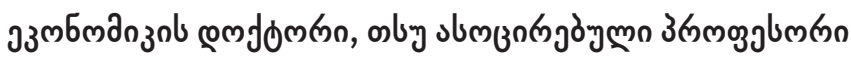

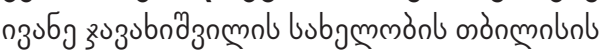

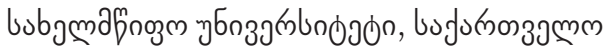

ia.natsvlishvili@tsu.ge

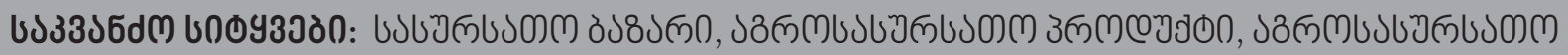

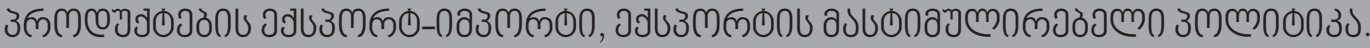

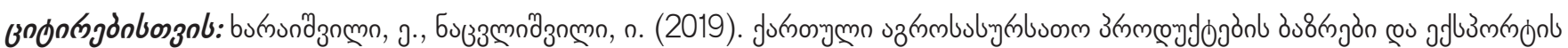

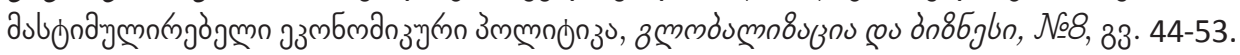

https://doi.org/10.35945/gb.2019.08.004

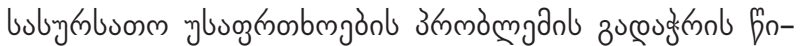

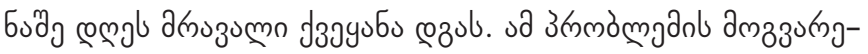

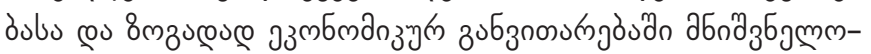

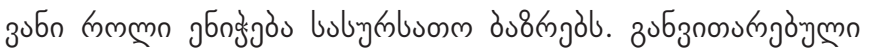

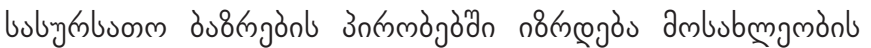

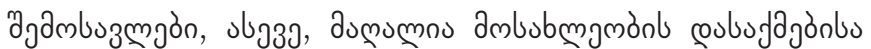

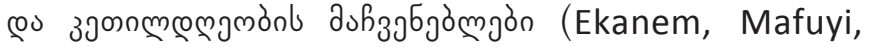

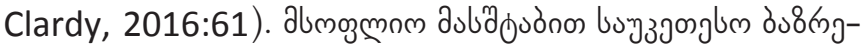

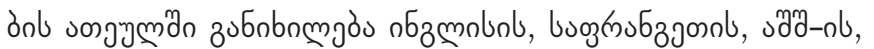

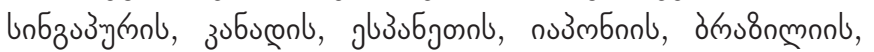
3gr

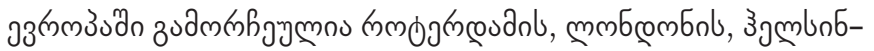

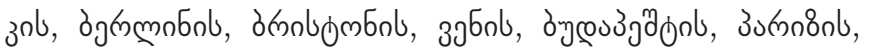

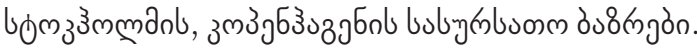

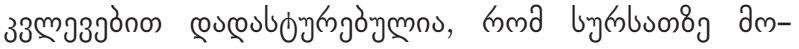

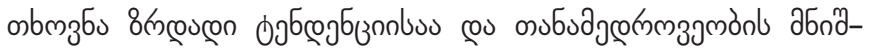

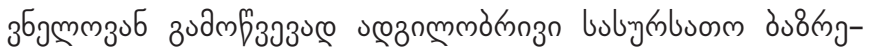
onb zubznoosungos zubnbnmgdo (Ekanem, Mafuya, Clardy,

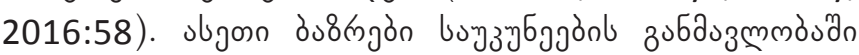

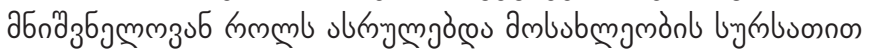

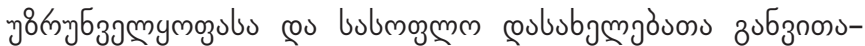

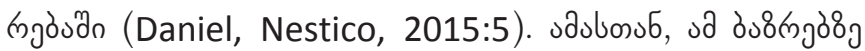

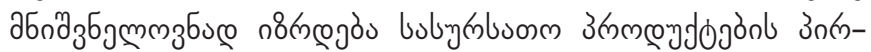

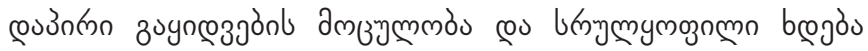

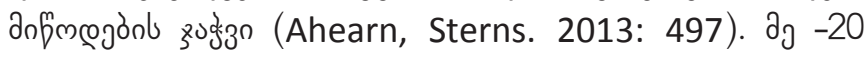

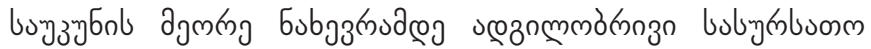

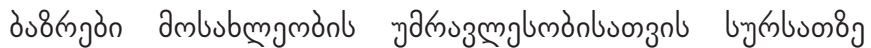

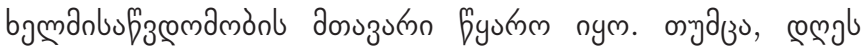

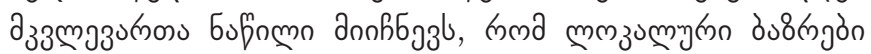

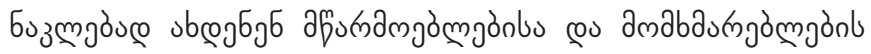

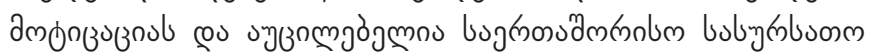

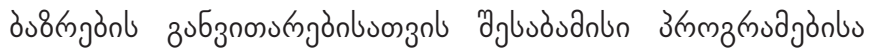
co उmmnonzol zgayduzgos (Martinez et al. 2010:35).

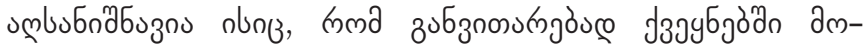

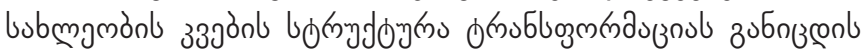

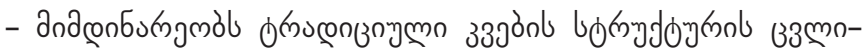

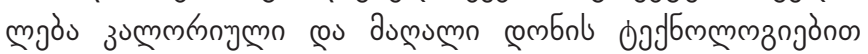

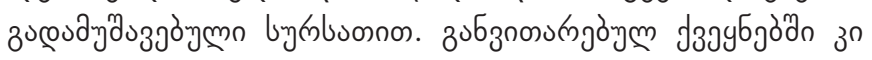

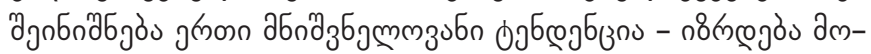

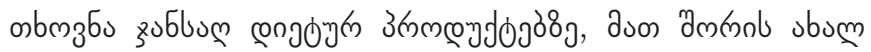




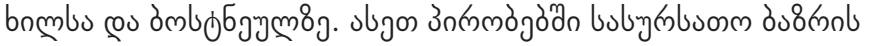

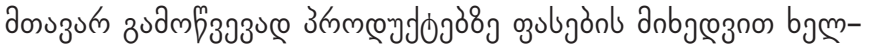

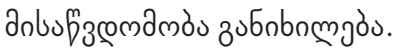

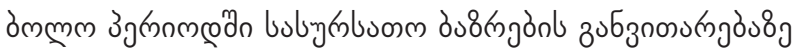

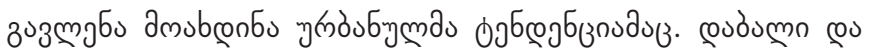

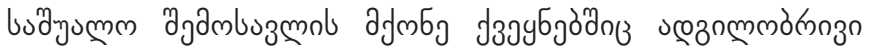

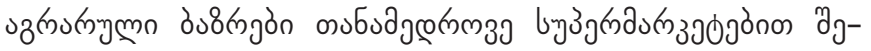

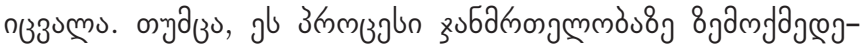

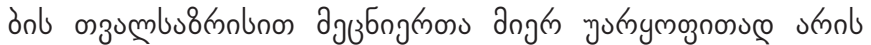

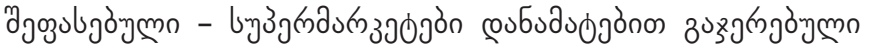

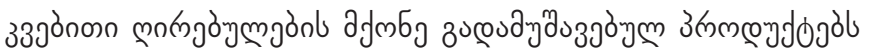

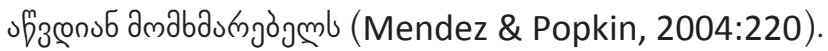

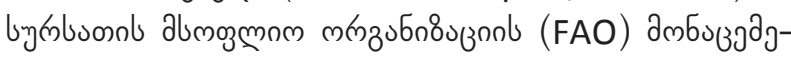

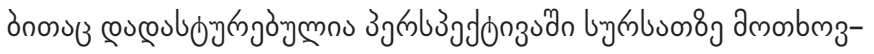

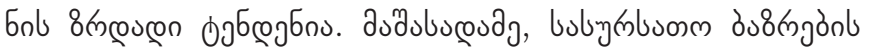

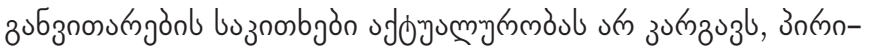

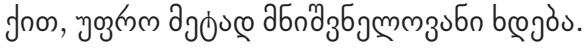

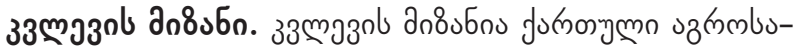

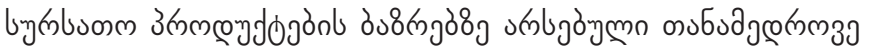

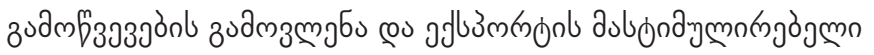

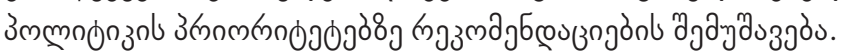

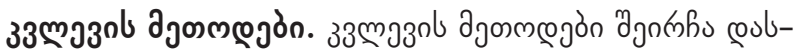

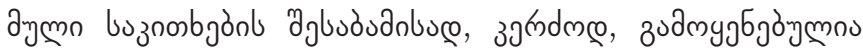

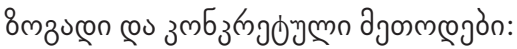

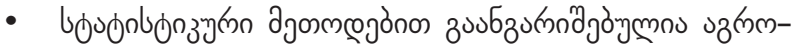

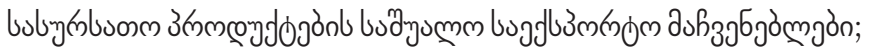

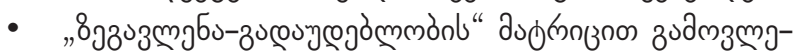

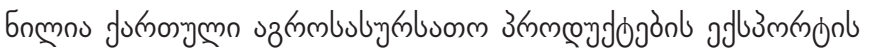

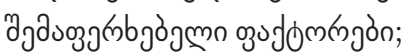

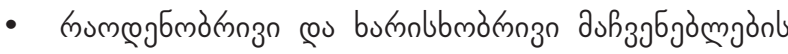

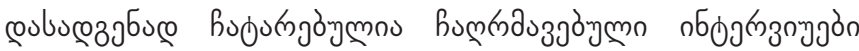

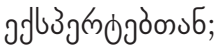

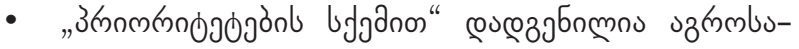

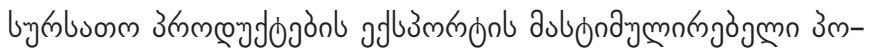

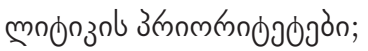

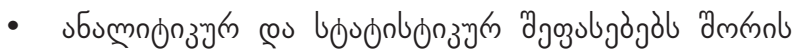

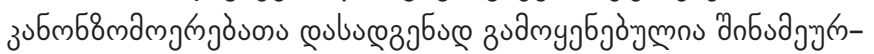

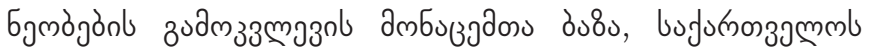

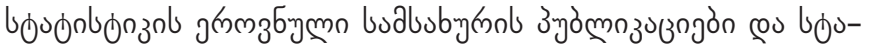

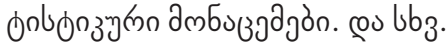

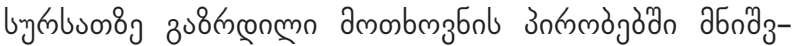

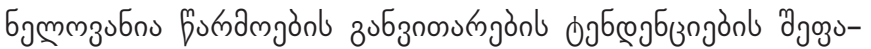

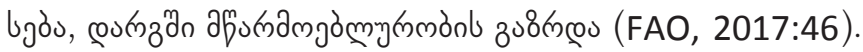

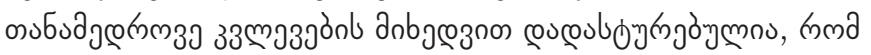

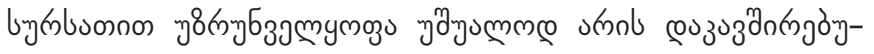

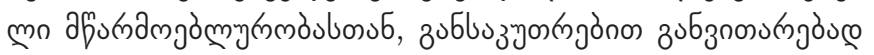
Jзэyбjةżn (Nsiah, Wallace, 2017).

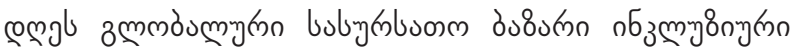

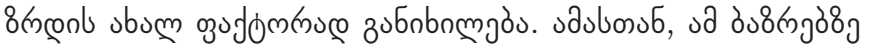

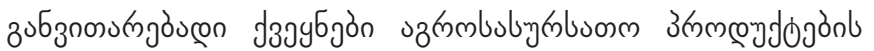

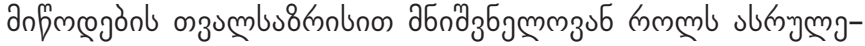
jəб (Dybowski, Bugala, 2016).

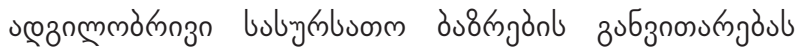

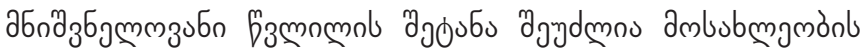

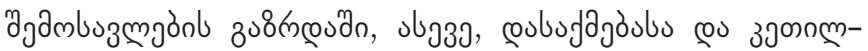

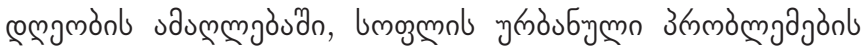
amz3urnjöodn (Ekanem et al., 2016).

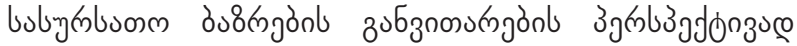

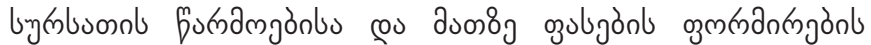

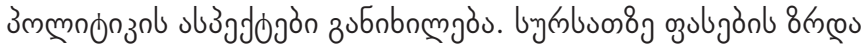

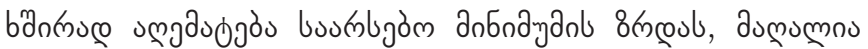

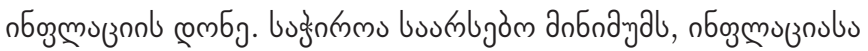

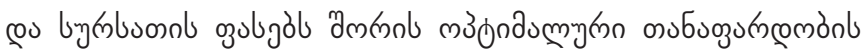

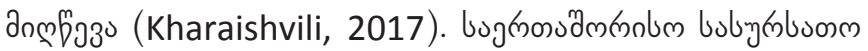

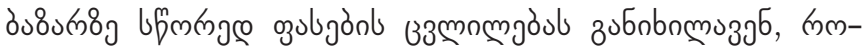

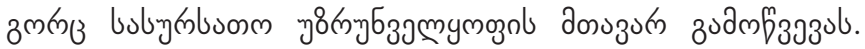

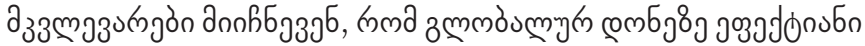

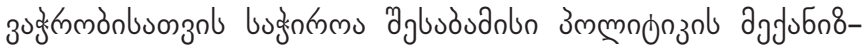

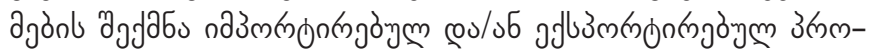

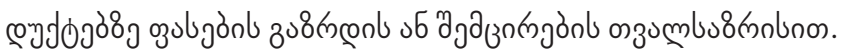

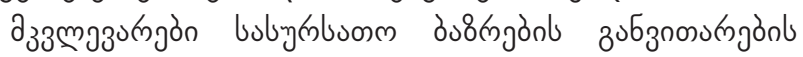

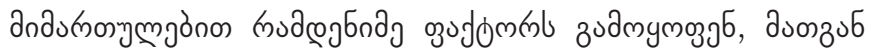

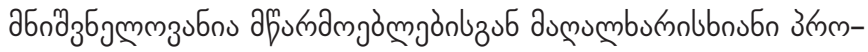

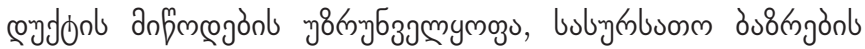

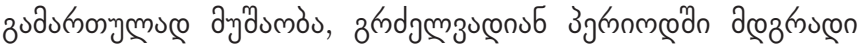

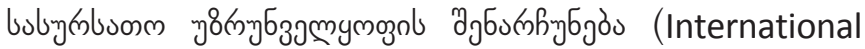
Panel of Experts on Sustainable Food Systems, 2015).

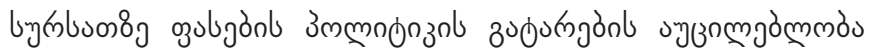

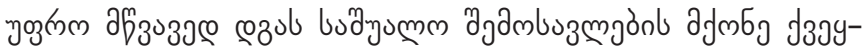

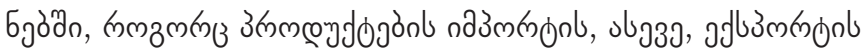

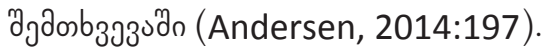

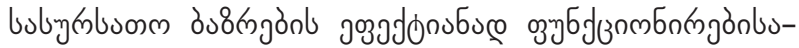

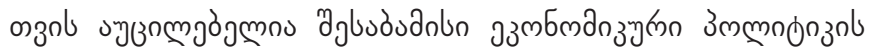

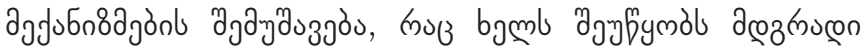

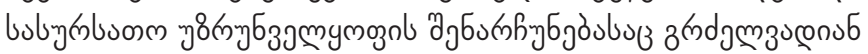

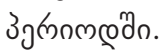

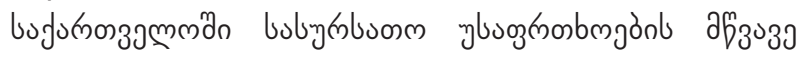

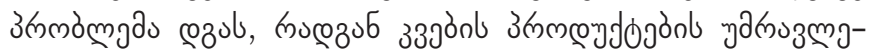

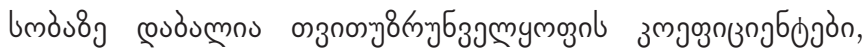

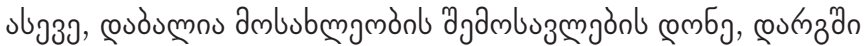

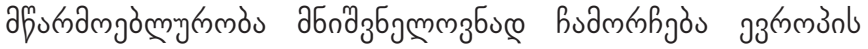

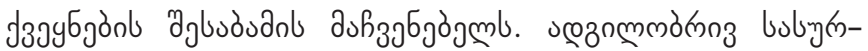

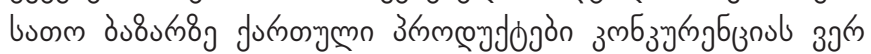

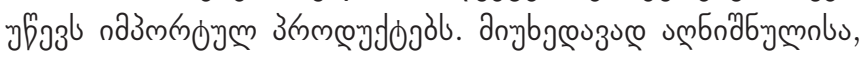

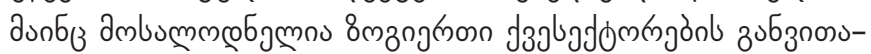

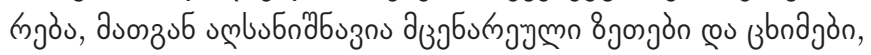
бmbыб उஙmmœyjdojòn (Ecorys, 2012:40).

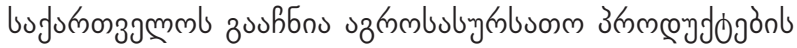

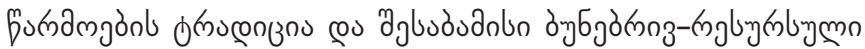




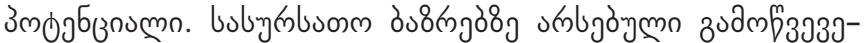

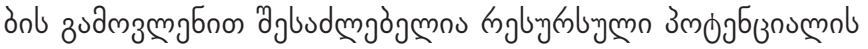

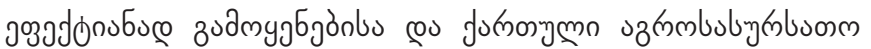

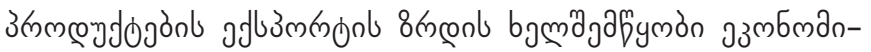

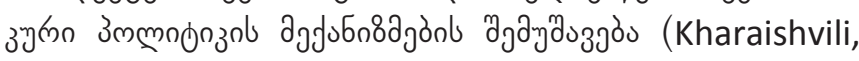
2018).

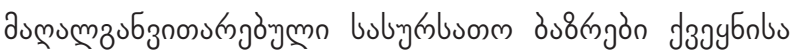

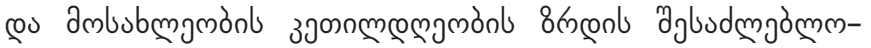

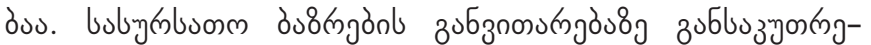

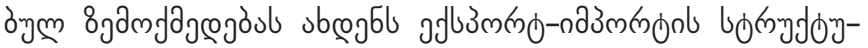

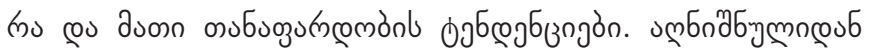

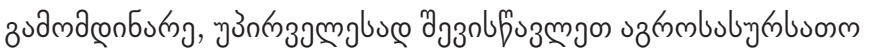

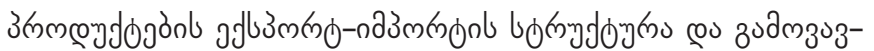

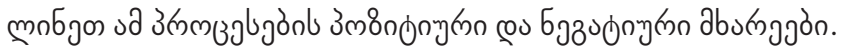

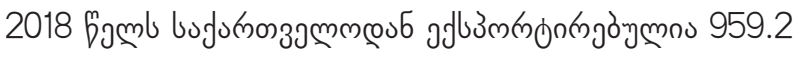

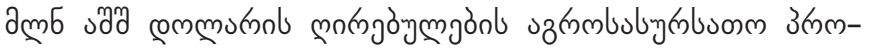

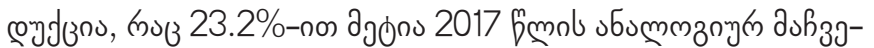

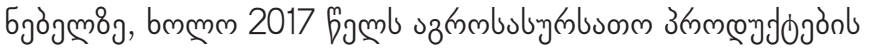

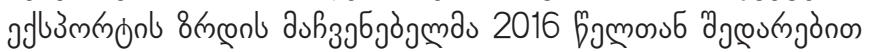
12.3\% गुง

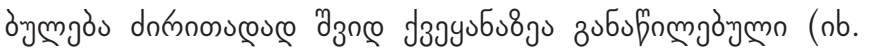
enuz(rods 1).

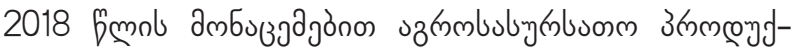

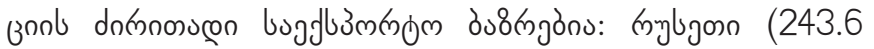
a

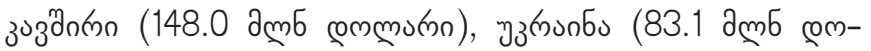

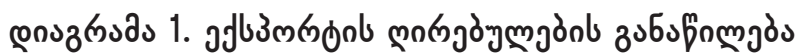

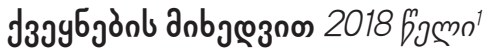

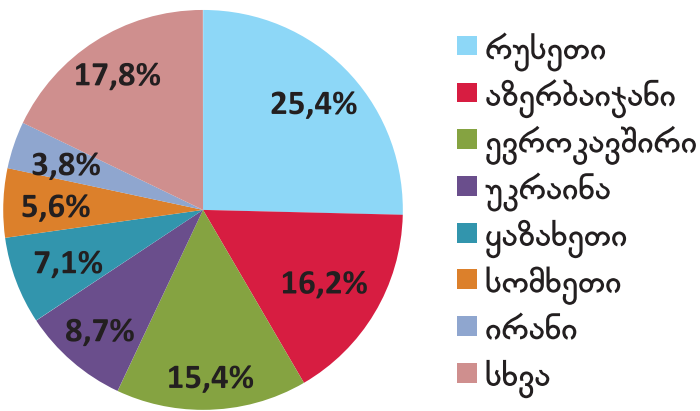

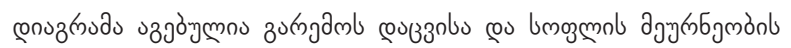

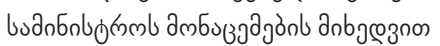

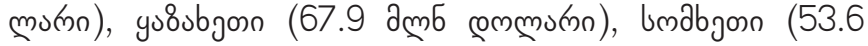

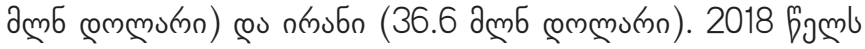

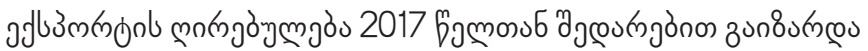

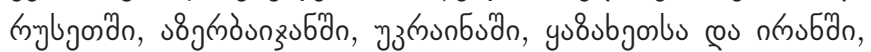

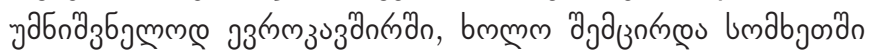

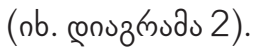

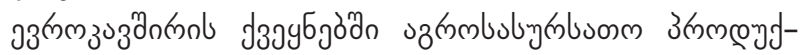

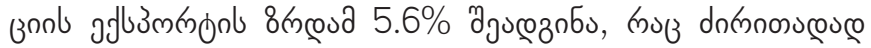

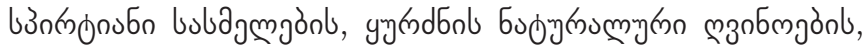

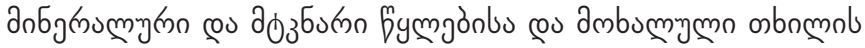

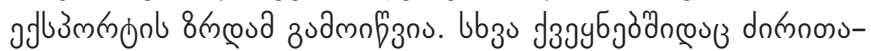

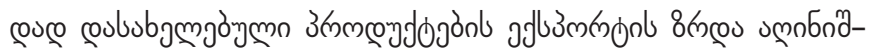
Ejò (nb. enszgrado 3).

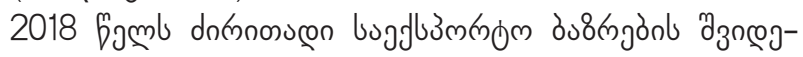

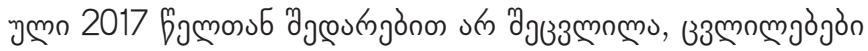

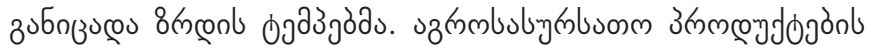

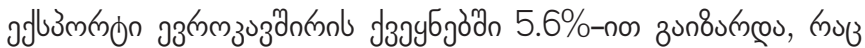

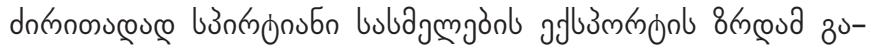

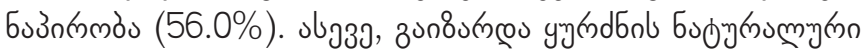

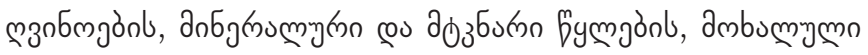

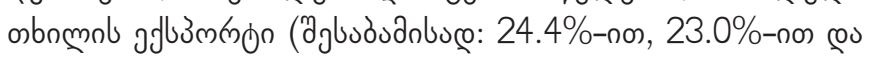

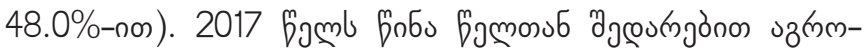

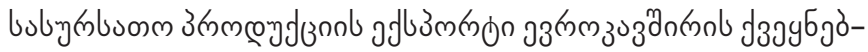

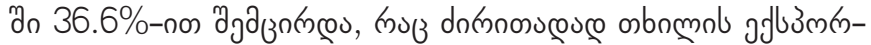

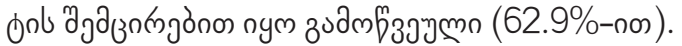

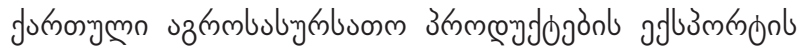
аง Ззаб

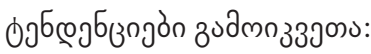

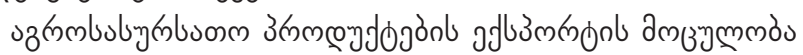

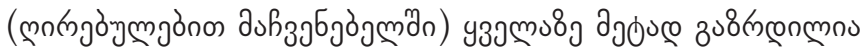

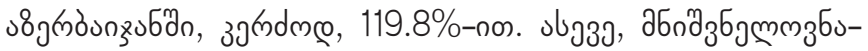

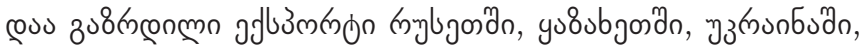

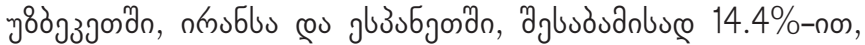
78.4\%-non, 24.7\%-non, 269.6\%-non, 36.8\%-non pu 167.6\%-

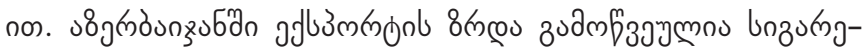

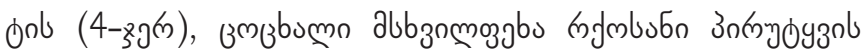

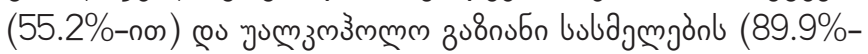

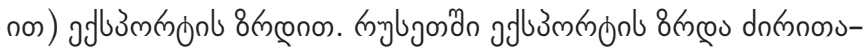

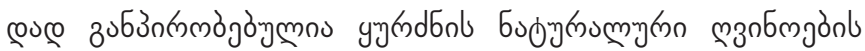

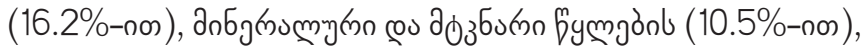

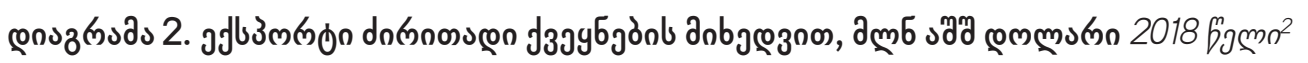

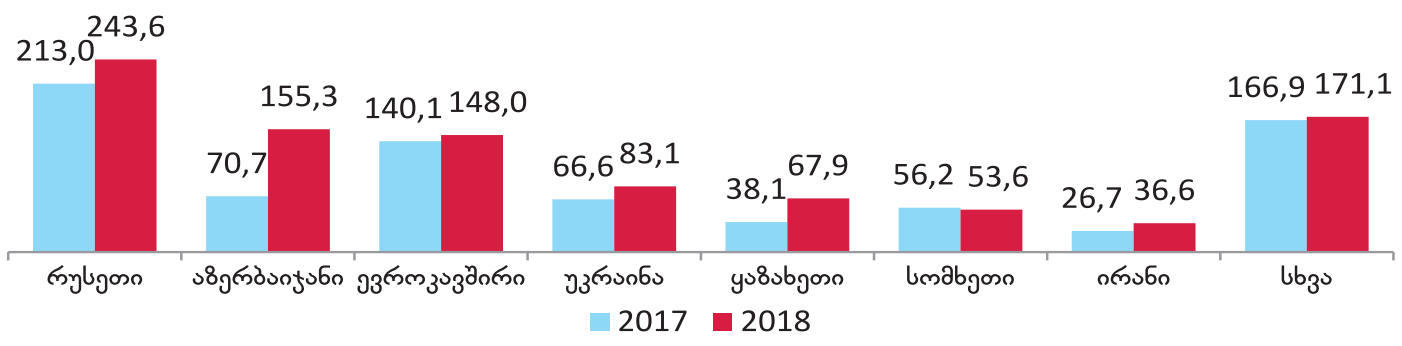

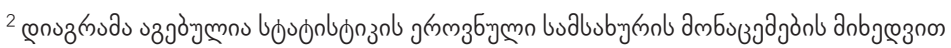




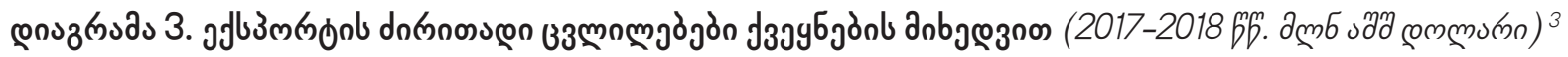

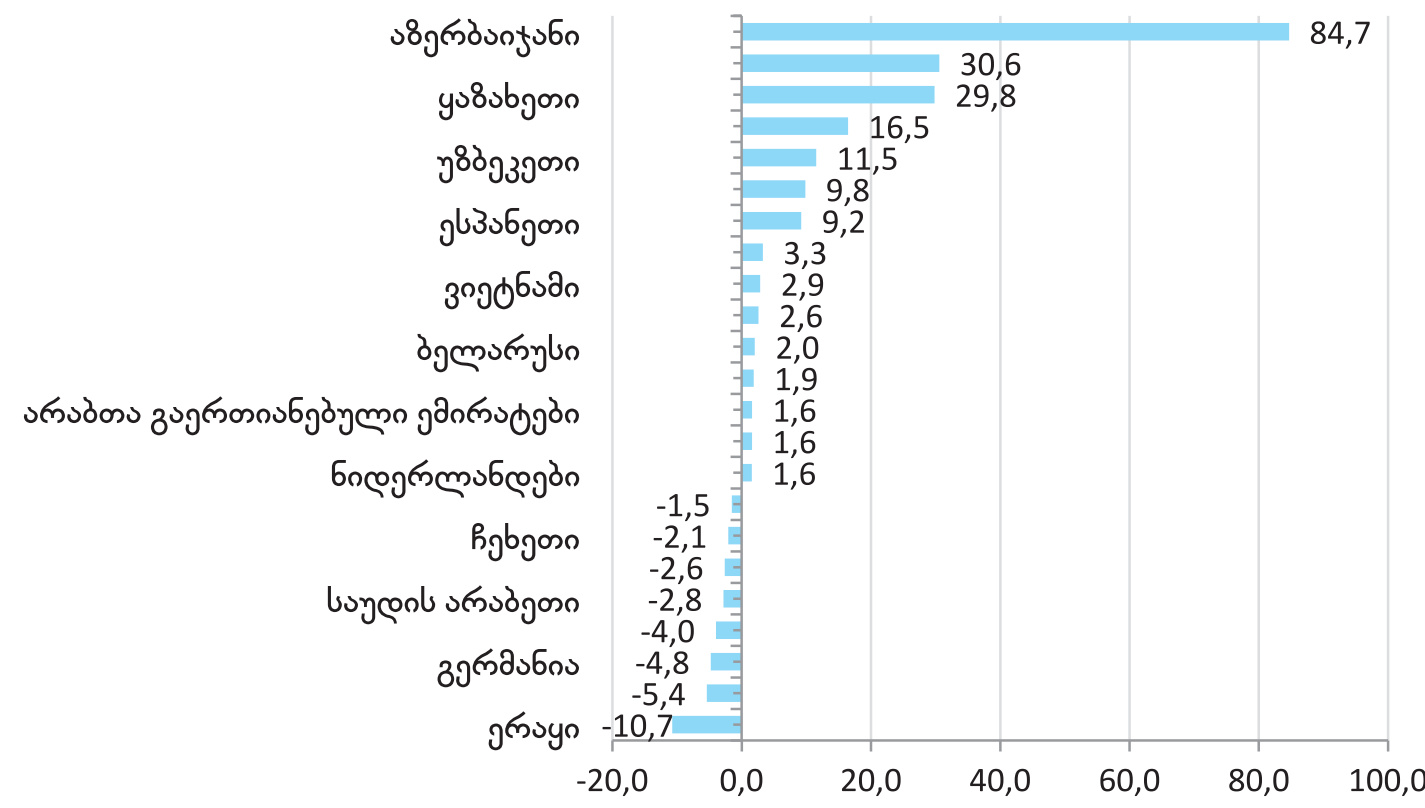

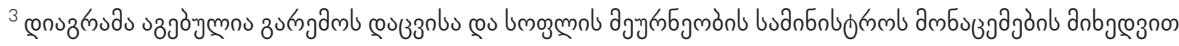

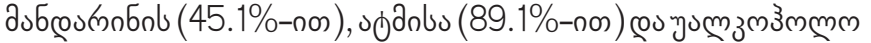

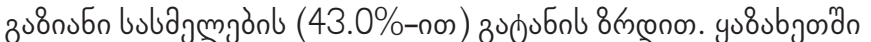

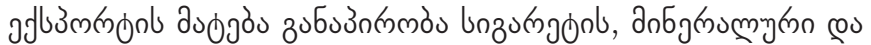

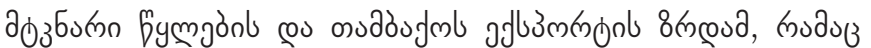

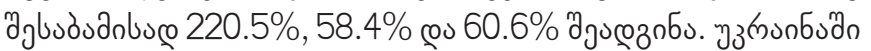

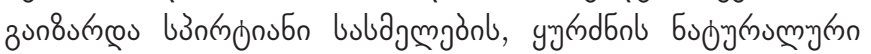

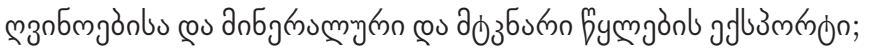

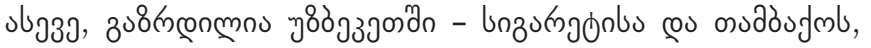

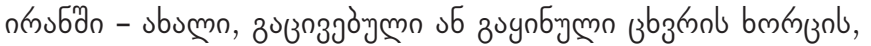

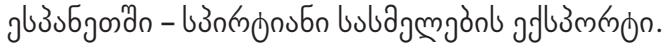

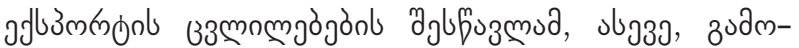

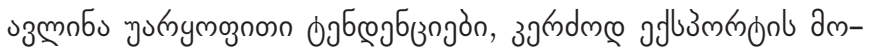

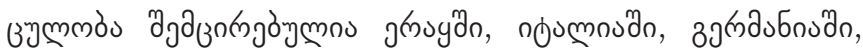

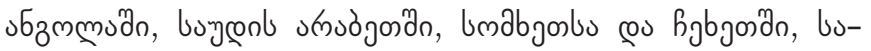

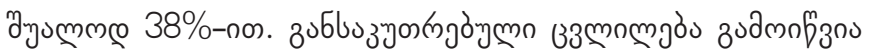

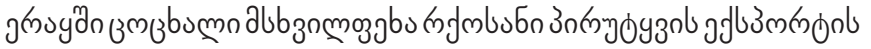

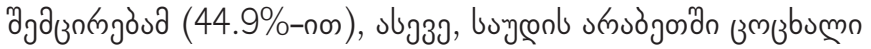

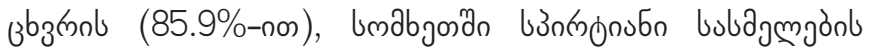

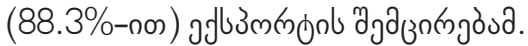

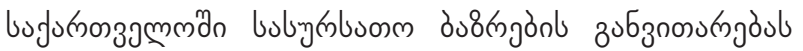

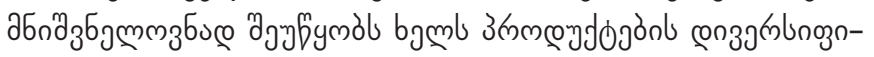

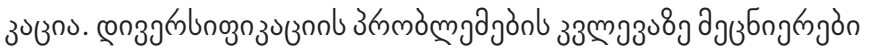

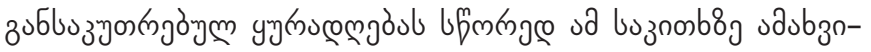

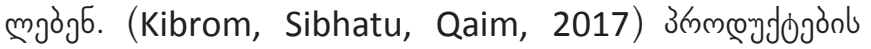

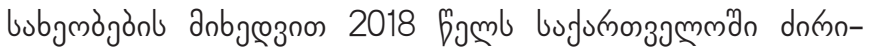

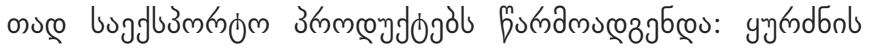

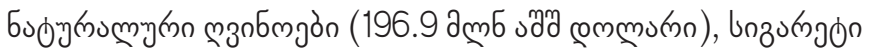

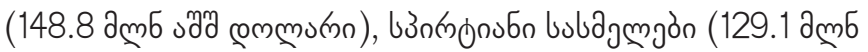

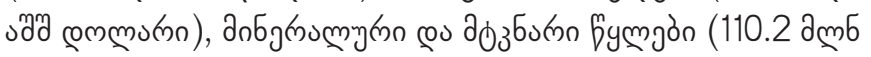

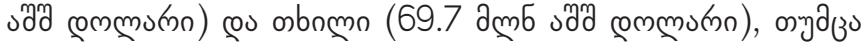

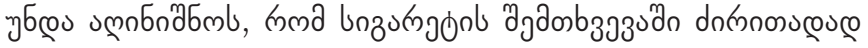

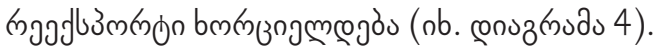

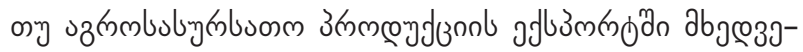

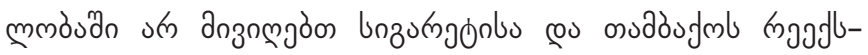

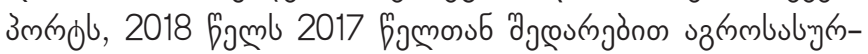

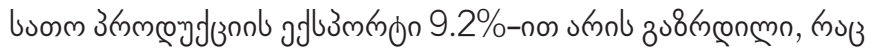

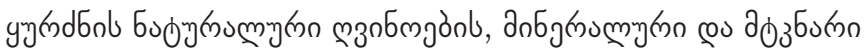

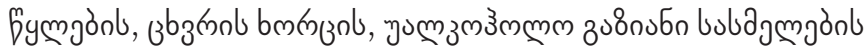

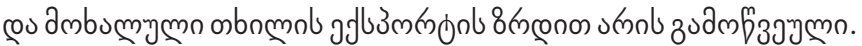

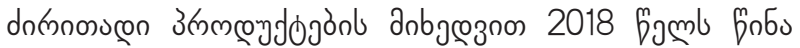

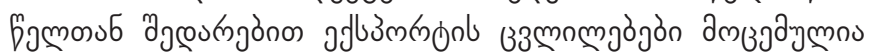

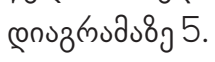

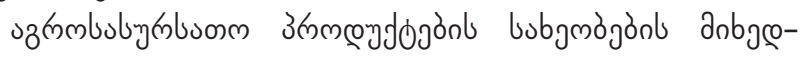

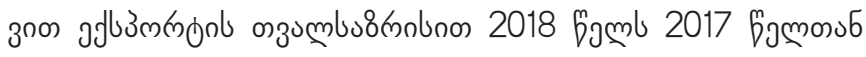

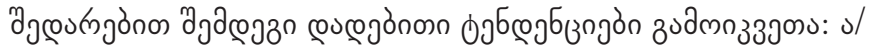

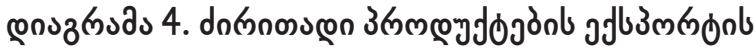

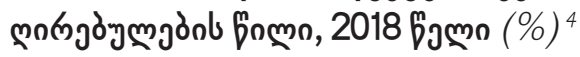

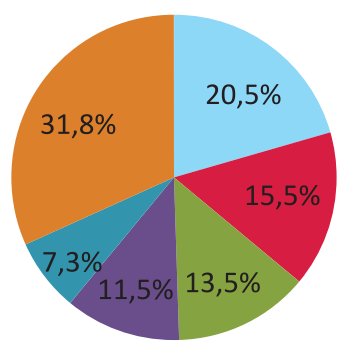

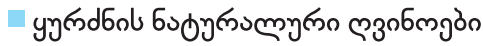

- unzurnodo

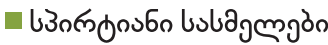

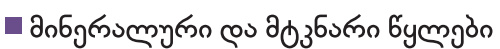

abomo

- ib3o

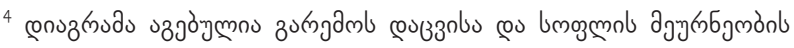

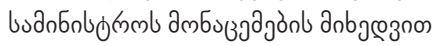




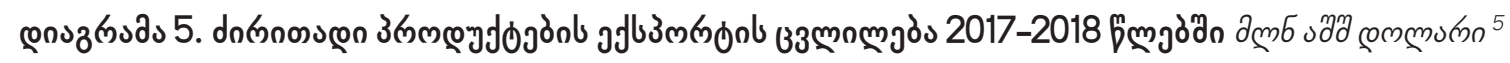

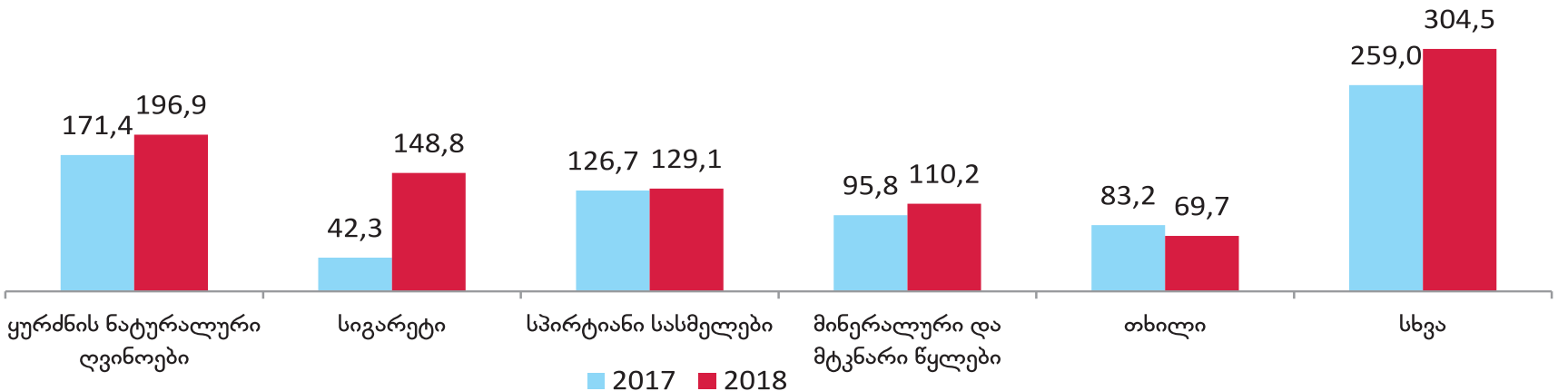

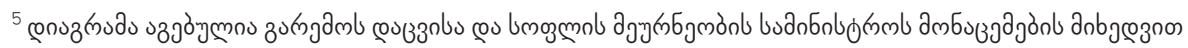

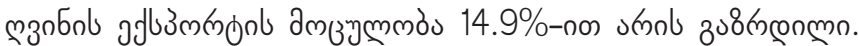

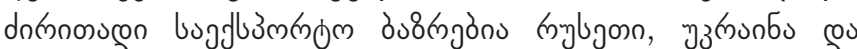

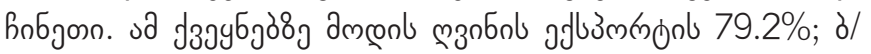

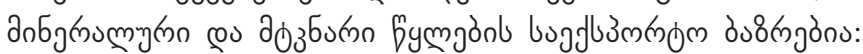

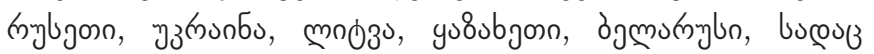

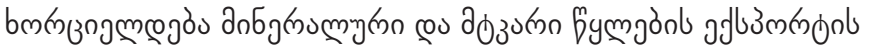

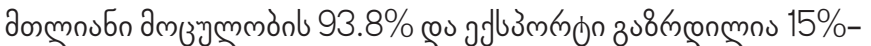

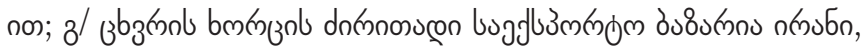

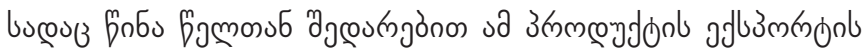

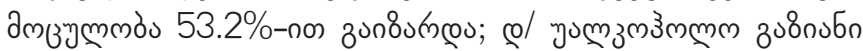

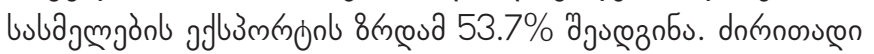

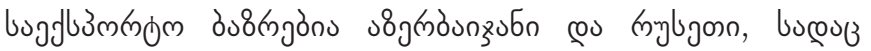

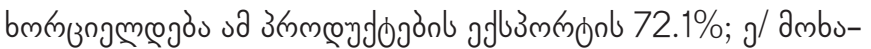

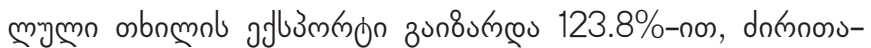

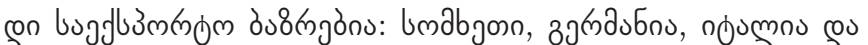

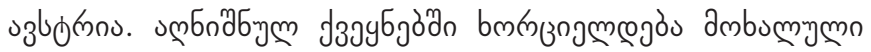

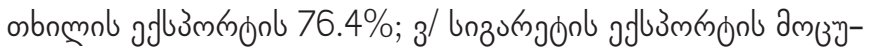

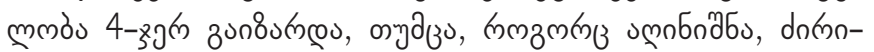

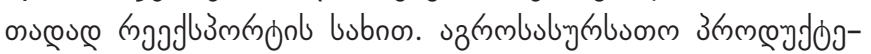

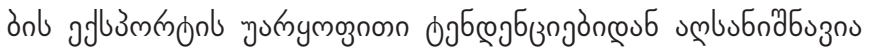

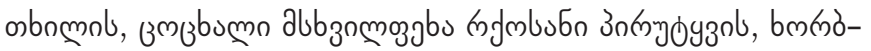

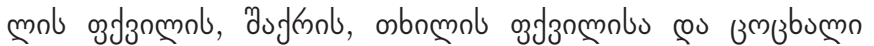

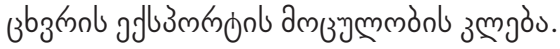

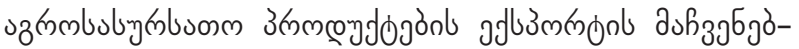

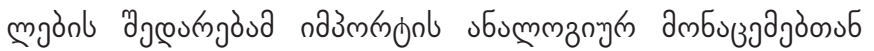

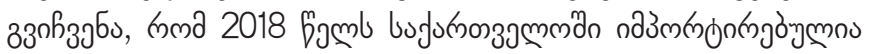

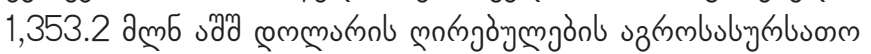

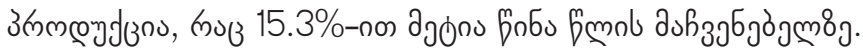

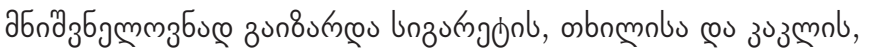

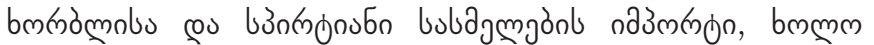

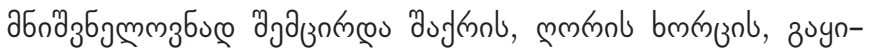

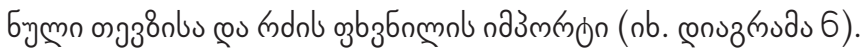

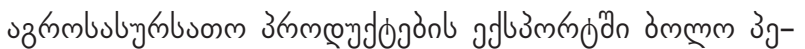

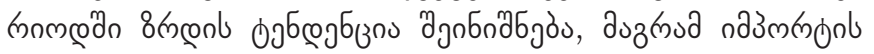

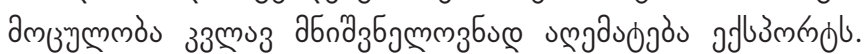

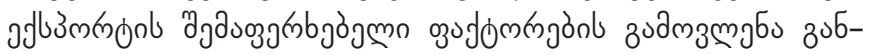

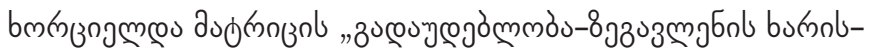
bnb" budyumgànon (nb. conuzmoas 7).

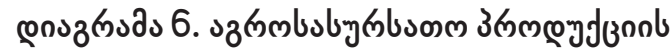

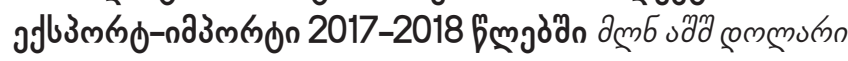

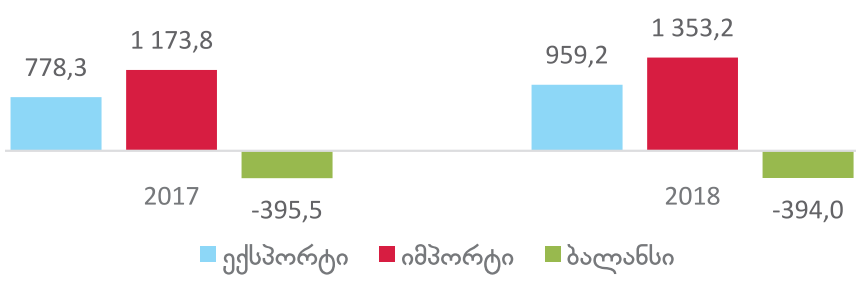

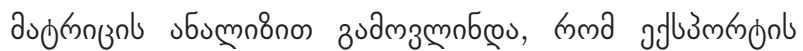

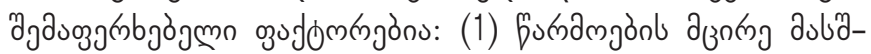

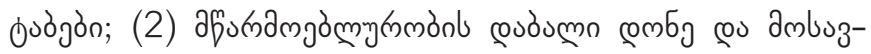

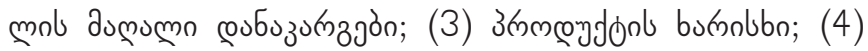

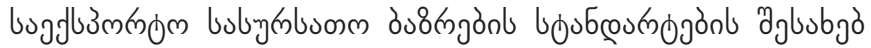

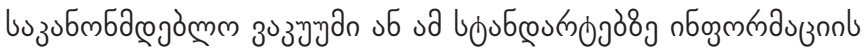

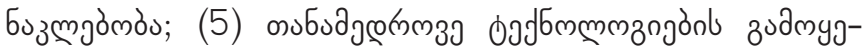

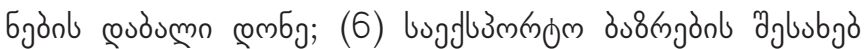

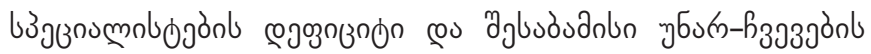

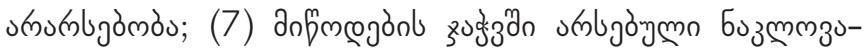
agajòn pou libzo.

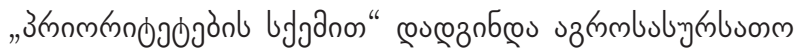

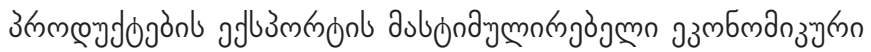

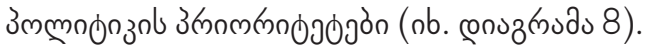

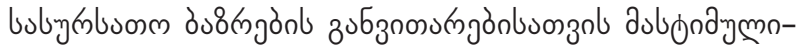

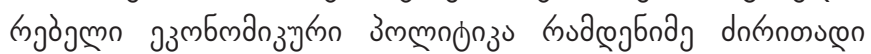

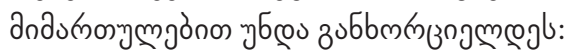

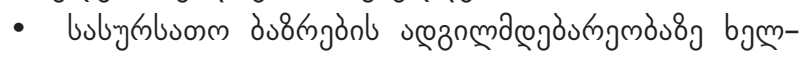

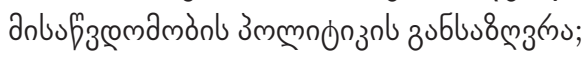

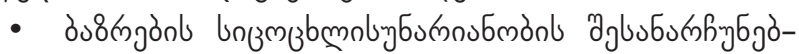

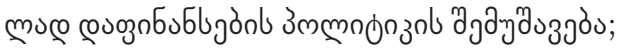

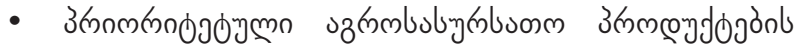

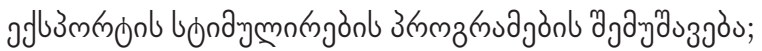

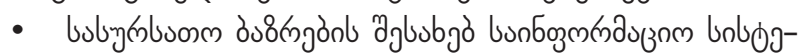

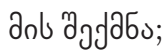

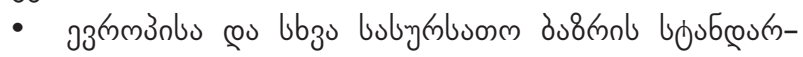

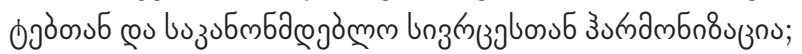

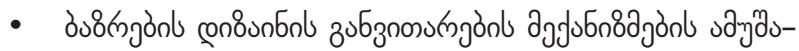
39ò cou bb3. 


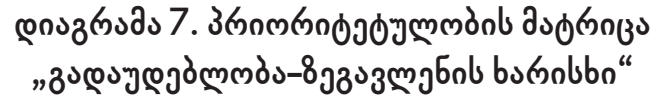

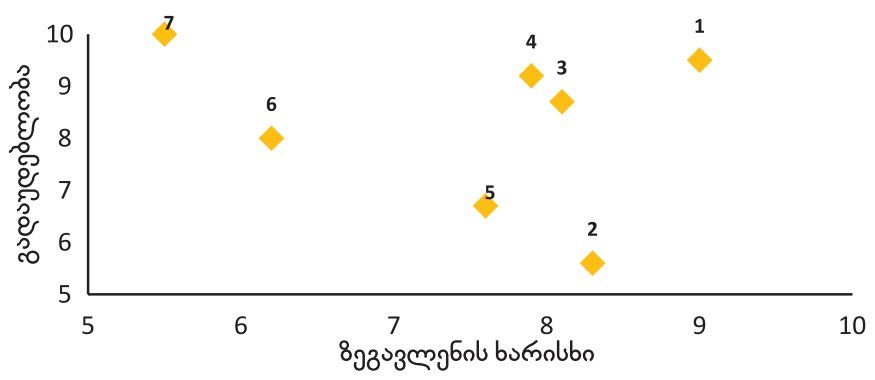

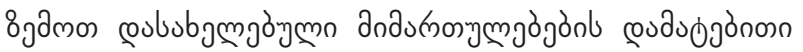

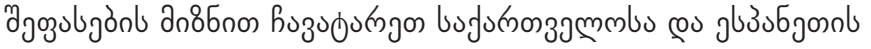

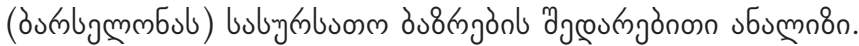

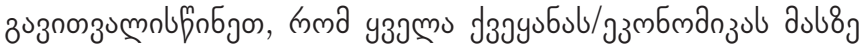

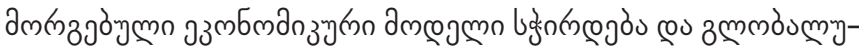

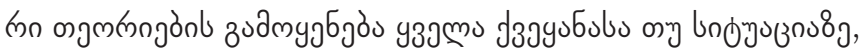

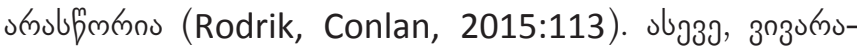

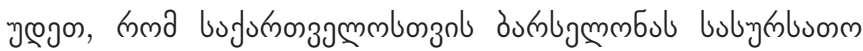

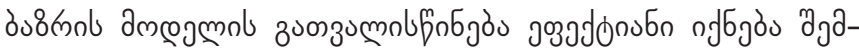

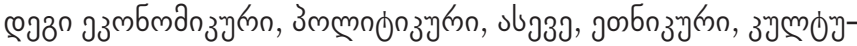

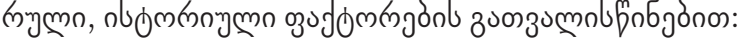

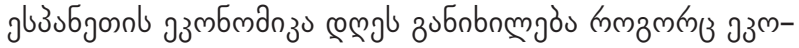

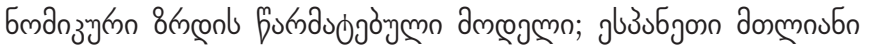

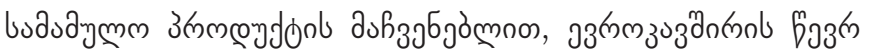

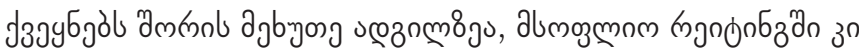

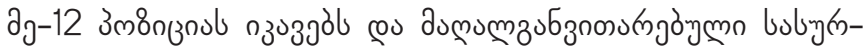
buonm bu8mgòn zushEns;

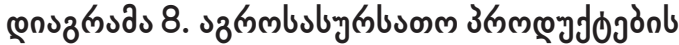

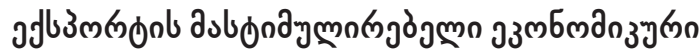

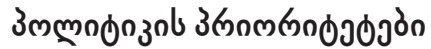

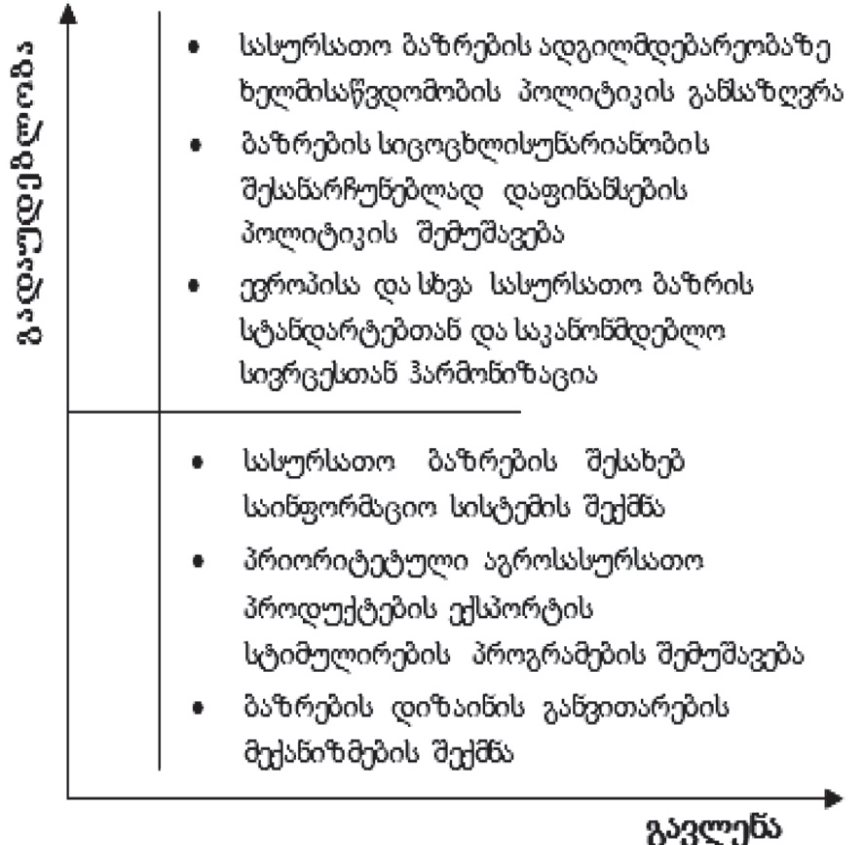




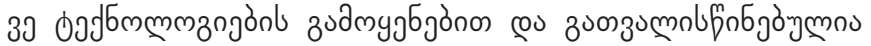

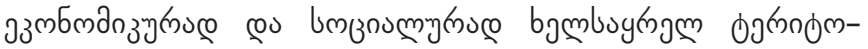

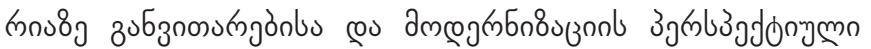

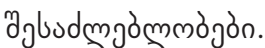

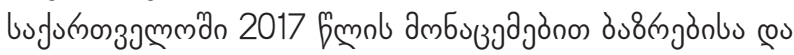

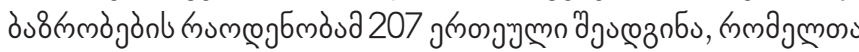

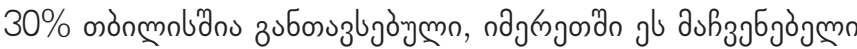

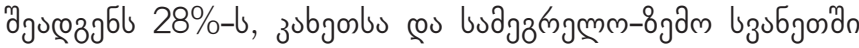

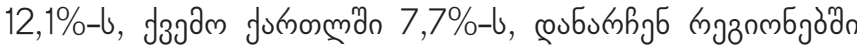

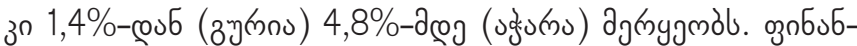

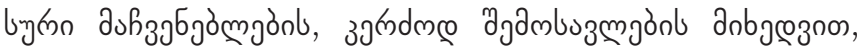

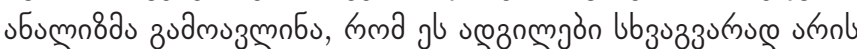

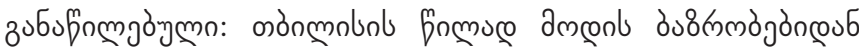

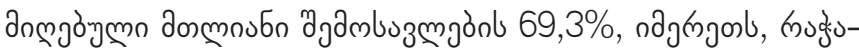

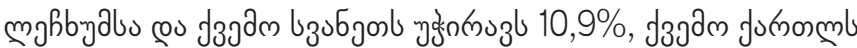

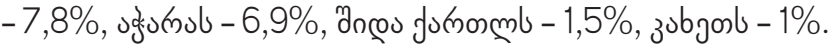

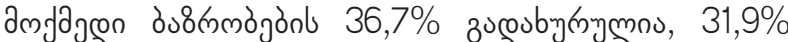

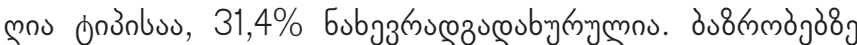

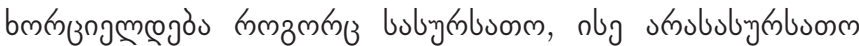

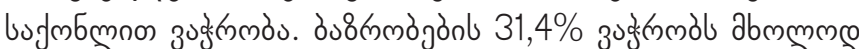

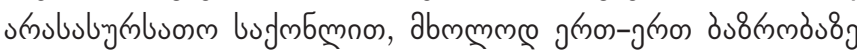

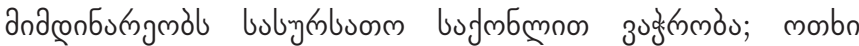

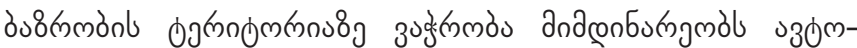

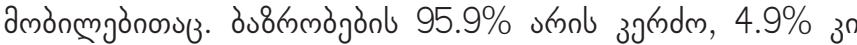

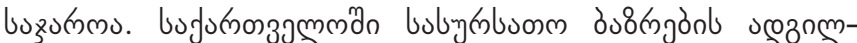

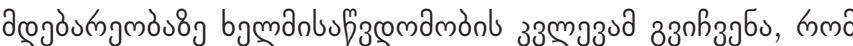

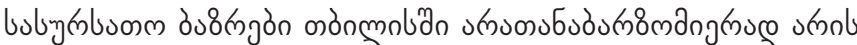

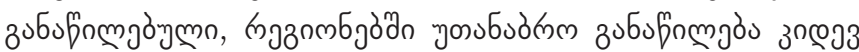

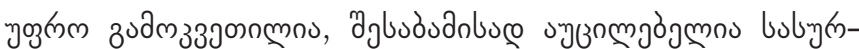

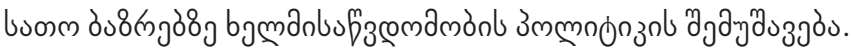

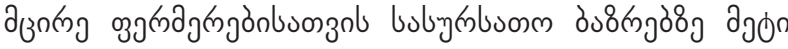

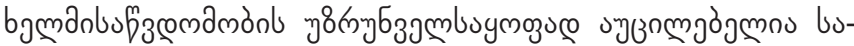

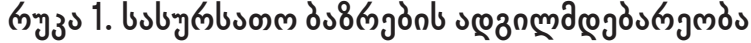

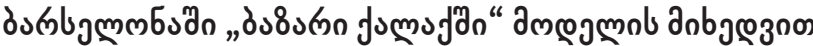

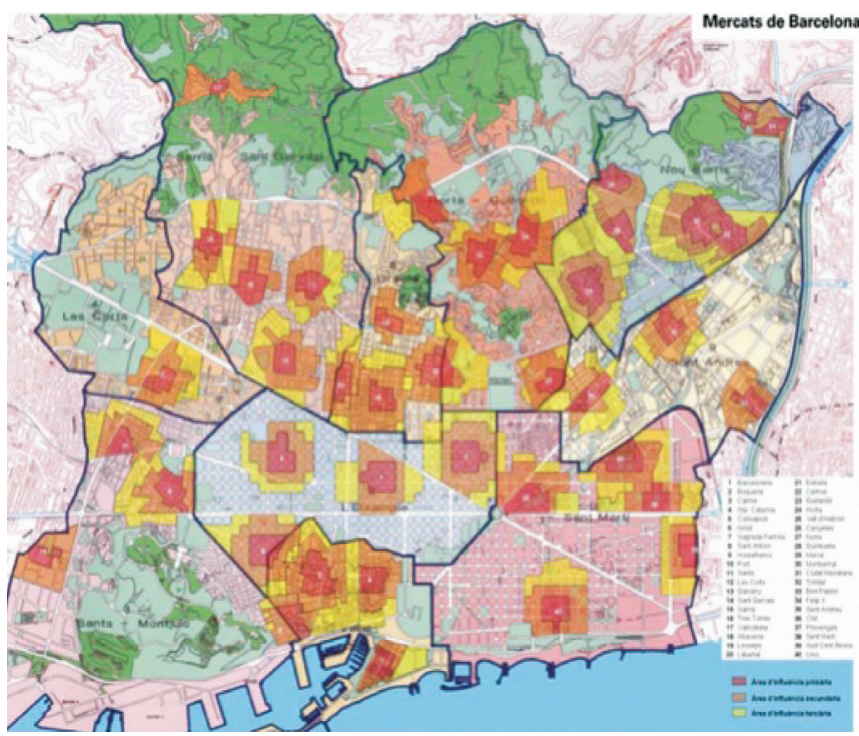

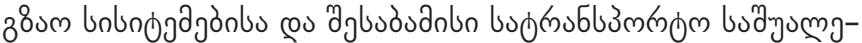

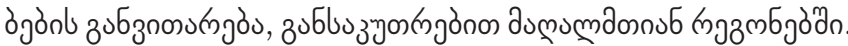

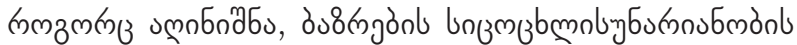

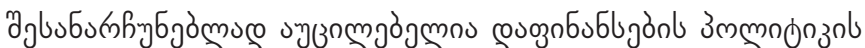

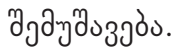

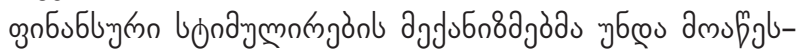

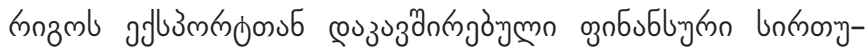

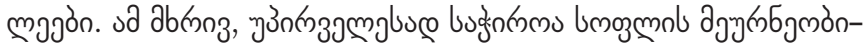

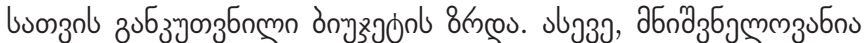

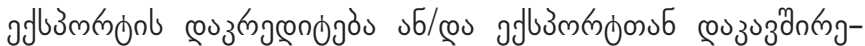

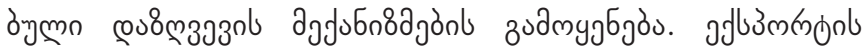

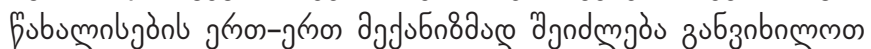

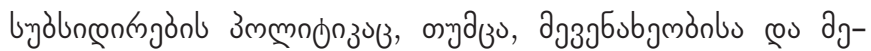

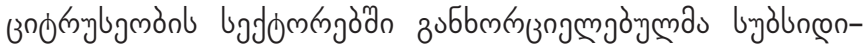

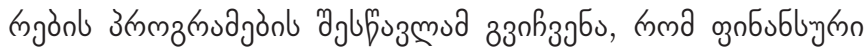

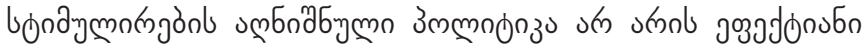

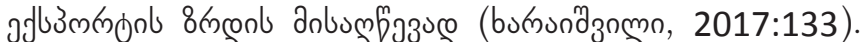

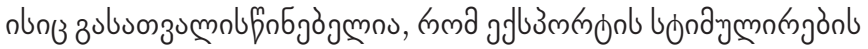

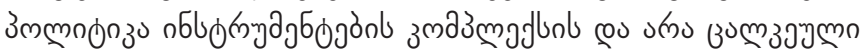

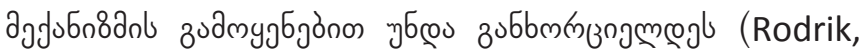
Conlan, 2015).

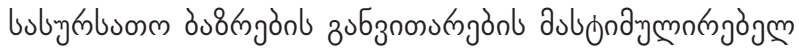

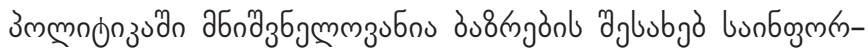

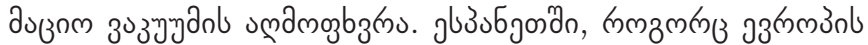

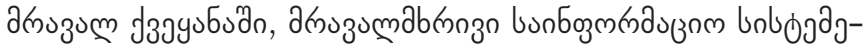

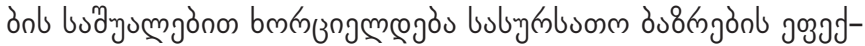

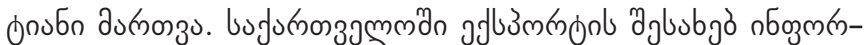

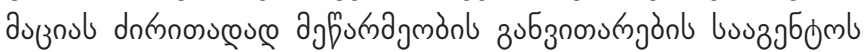

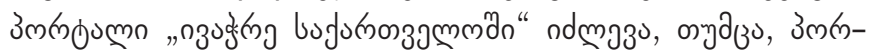

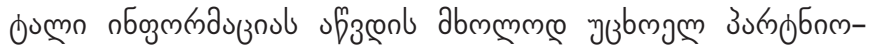

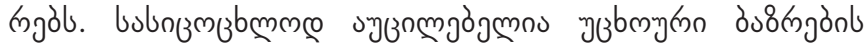

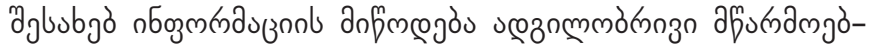
mgànlonzoluos.

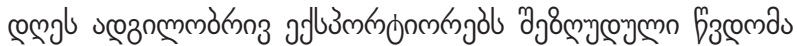

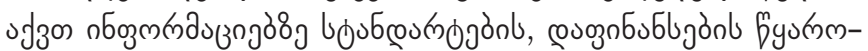

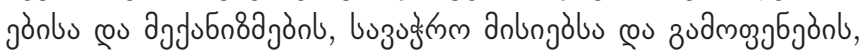

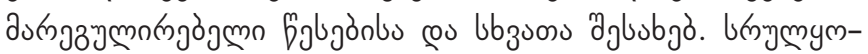

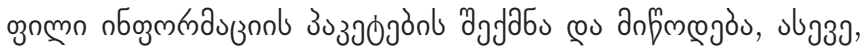

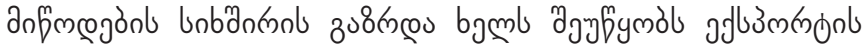

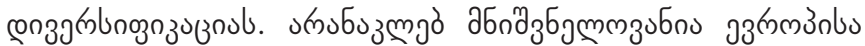

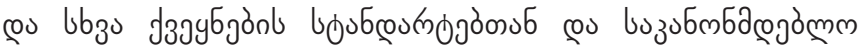

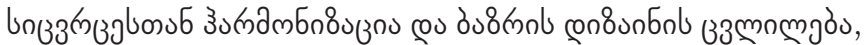

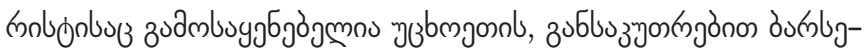

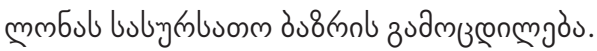

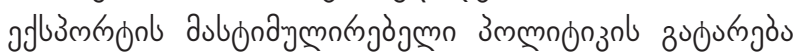

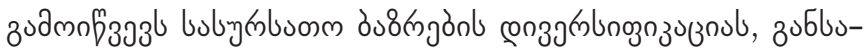

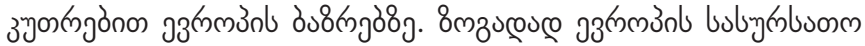

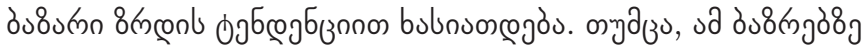

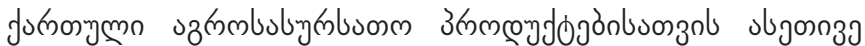

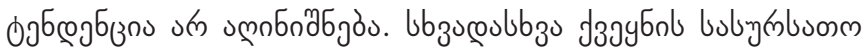




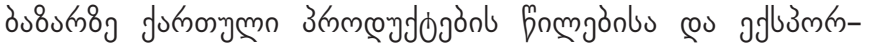

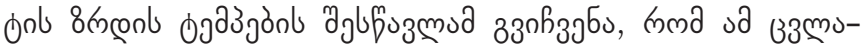

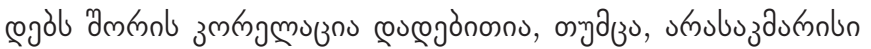

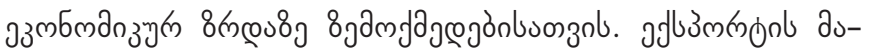

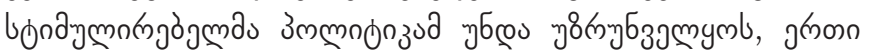

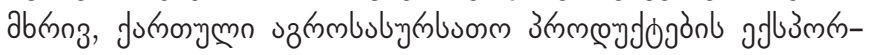

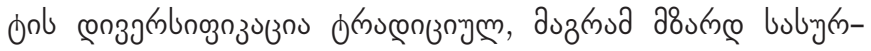

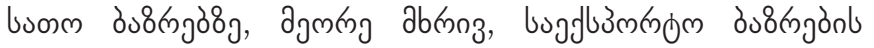

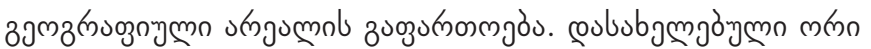

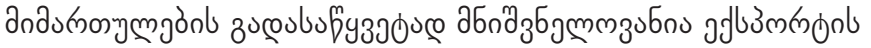

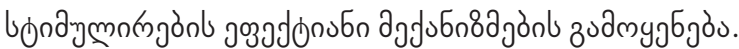

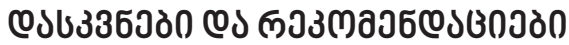

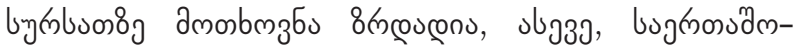

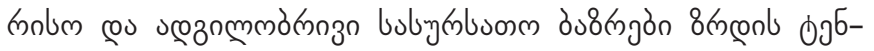

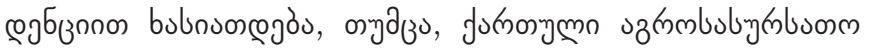

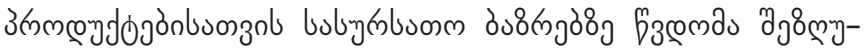

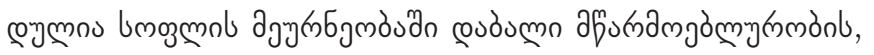

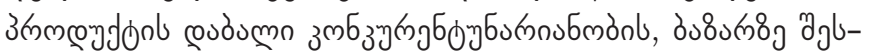

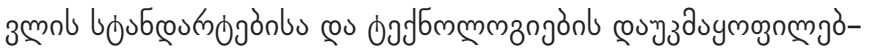

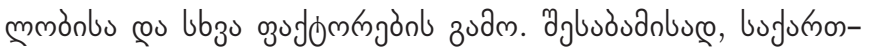

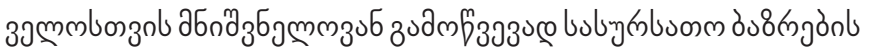

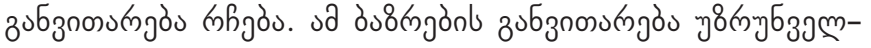

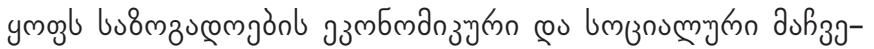

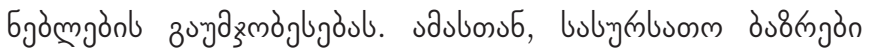

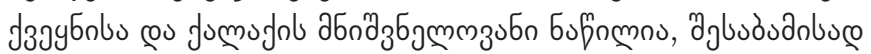

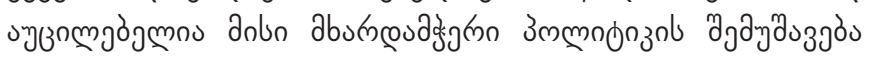

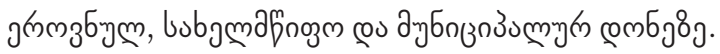

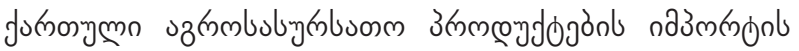

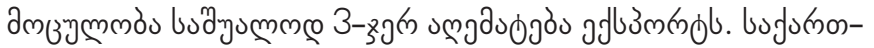

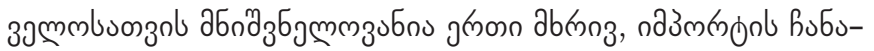

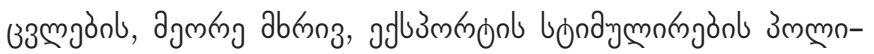
onzols zojurnjos.

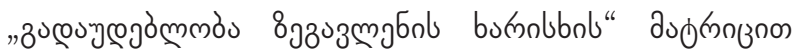

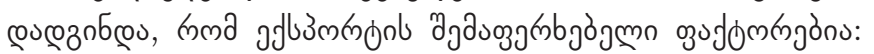

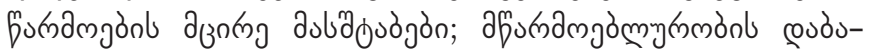

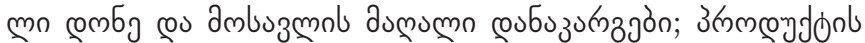

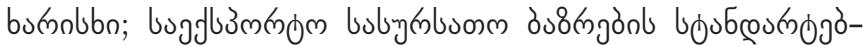

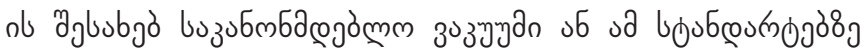

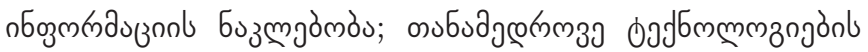

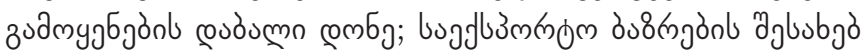

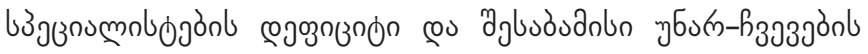

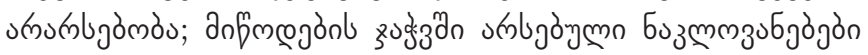
goubbas.

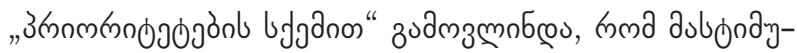

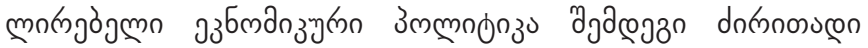

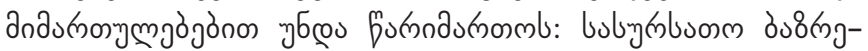

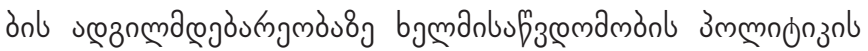

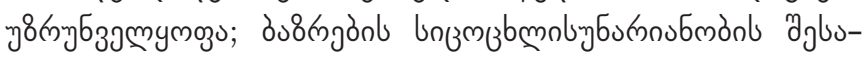

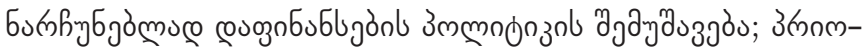

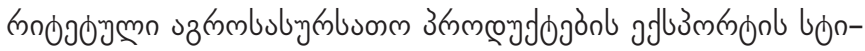

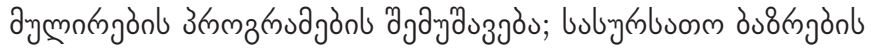
चgbubjo bunбogm

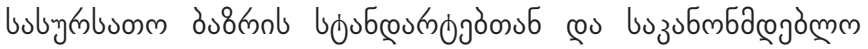

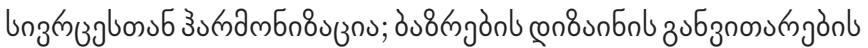

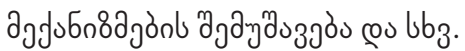

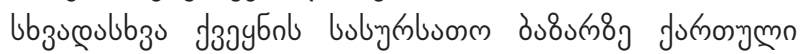

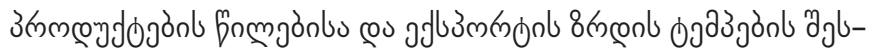

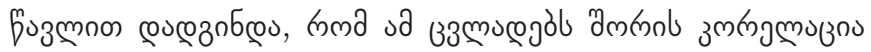

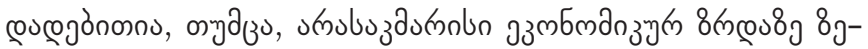

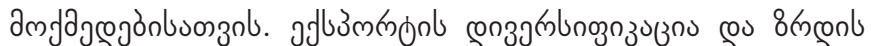

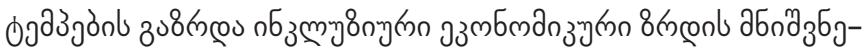

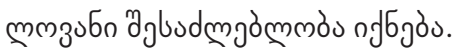

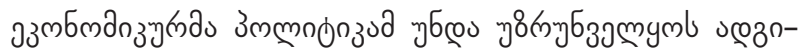

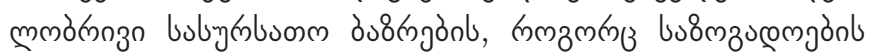

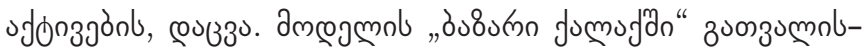

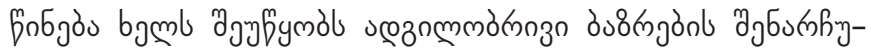

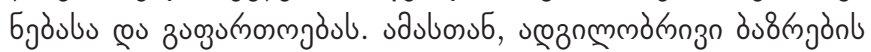

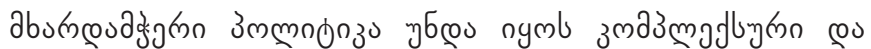

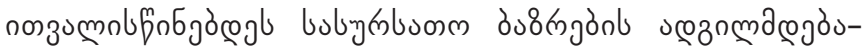
mgmònl, @usogn

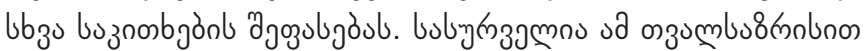

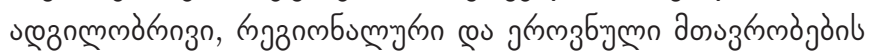

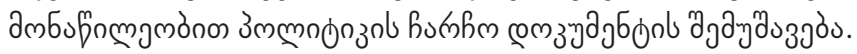

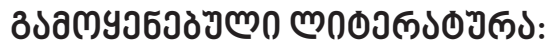

1. Kharaishvili E., (2017). Wine Market and Competitive Models oF the Viticulture-Winemaking Industry in Georgia [Ghvinis bazari da mevenakheoba-maghvineobis konkurentuli modelebi Sakartveloshi]. Tbilisi, pp.133 (In Georgian).

2. Andersen P. (2014). Food price Policy in an Era of Market Instability (A Political Economy Analisis).

3. Ahearn, M., Sterns J. (2013). "Direct-to-consumer sales of farm products: producers and supply chains in the southeast." Journal of Agricultural and Applied Economics 45(3).

4. Belloc, M. Di Maio, M. (2011). Survey of the Literature on the successful strategies and practices for export promotion by developing countries.

5. Daniel K., Nestico S. (2015). Policies that Support Local Fresh Food Markets, International Examples.

6. Dybowski G., Bugala A. (2016). GLOBAL FOOD MARKET-NEW FACTORS INFLUENCING DEVELOPMENT. AGROFOR International Journal, Vol. 1, Issue No. 2. 
7. Ecorys, (2012), Trade Sustainability Impact Assessment in support of negotiations of a DCFTA between the EU and Georgia and the Republic of Moldova.

8. Ekanem E., Mafuyai M., Clardy A. (2016). Economic Importance of Local Food Markets: Evidence from the Literature.

9. International Panel of Experts on Sustainable Food Systems (2015). The New Science of Sustainable Food Systems Overcoming Barriers to Food Sustems Reform.

10. Kharaishvili E., (2017), DIRECTIONS FOR IMPROVING THE SUPPLY CHAIN IN THE AGRO-FOOD SECTOR OF GEORGIA. In Foresight management: formation and transformation adaptive business organizations. International collective monograph. Volume 2. Section 6.: 6.2. p. 268.

11. Kharaishvili E., (2018), Diversification of Agribusiness and Rural Development Models in Georgia. Kharaishvili, E., Gechbaia B., Mamuladze G. (2018), VEGETABLE MARKET: COMPETITIVE ADVANTAGES OF GEORGIAN PRODUCT AND COMPETITION CHALLENGES, Innovative Marketing, Volume 14, Issue 3.

12. Kharaishvili E., Erkomaishvili G., Chavleishvili M., (2019), Trends of Agro-Food Production and Export Stimulating Economic Policy in Georgia, International Journal of Economics and Management Engineering, Vol:13, No:1.

13. Kharaishvili E., Lazariashvili T., Natsvlishvili I., (2019), GLOBAL EXPERIENCE OF BIO PRODUCT MARKET AND ITS CHALLENGES IN GEORGIA IN THE CONTEXT OF SUSTAINABLE WELL-BEING.

14. Kibrom T., Sibhatu, Matin Qaim (2017). Rural food security, subsistence agriculture and seasonality.

15. Martinez S. , Hand M., Da Pra M., Pollack S., Ralston K., Smith T., Vogel S., Clark S., Lohr L., Low S. , and Newman C. (2010). Local Food Systems Concepts, Impacts, and Issues. A Report from the Economic Research Service.

16. Michelle A. Mendez, Barry M. Popkin (2004). The Electronic Journal of Agricultural and Development Economics, 2004, vol. 1 , issue 2 .

17. Nsiah C., Wallace B., (2017).Trends in Agricultural Production Efficiency and Its Implications for Food Security in SubSaharan African Countries.

18. Rodrik D., Conlan J. (2015), Economics Rules: The Rights and Wrongs of The Dismal Science.

19. FAO (2017). The future of food and agriculture - Trends and challenges.

20. Top 10 Food Markets in Europe [Evropis 10 sauketeso sasursato bazari], (In Georgian). https://www.theguardian.com/ travel/2014/dec/01/top-10-food-markets-europe-berlin-vienna-london

21. Top 10 Food Markets in World [Msoflios 10 sauketeso sasursato bazari], (In Georgian). https://foodtank.com/ news/2014/11/ten-fantastic-food-markets/

22. National Statistics Office of Georgia, Export [ Saqartvelos statistikis erovnuli samsaxuri, exporti 2000-2016], (In Georgian). http://geostat.ge/?action=page\&p_id=136\&lang=geo

23. Entrepreneurship Development Agency of Georgia: Mission and Vission [Sakartvelos mewarmeobis ganvitarebis saagento, misia da khedvebi], (In Georgian).http://enterprisegeorgia.gov.ge/ge/aboutus/Mission--Vission

24. We and the Basques [ Chven da Baskebi], (In Georgian).http://www.georoyal.ge/?MTID=6\&TID=54\&id=138 


\title{
MARKETS OF GEORGIAN AGRO-FOOD PRODUCTS AND EXPORT STIMULATING ECONOMIC POLICY
}

\author{
ETERI KHARAISHVILI \\ Doctor of Economic Sciences, Professor \\ Ivane Javakhishvili Tbilisi State University, Georgia \\ Vice-Prezident of Academy of Economic \\ Sciencies of Georgia, Georgia \\ eter.kharaishvili@tsu.ge
}

\section{IA NATSVLISHVILI}

Candidate of Economic Sciences, Associate Professor

Ivane Javakhishvili Tbilisi State University, Georgia

ia.natsvlishvili@tsu.ge

\section{KEYWORDS: FOOD MARKET, AGRO-FOOD PRODUCTS, EXPORT-IMPORT OF AGRO-FOOD PRODUCTS, EXPORT} STIMULATING POLICY.

For citation: Kharaishvili, E., Natsvlishvili, I. (2019). Markets of Georgian Agro-Food Products and Export Stimulating Economic Policy, Globalization And Business, №8, pp. 44-53. https://doi.org/10.35945/gb.2019.08.004

\section{SUMMARY}

The article substantiates the importance of the development of food markets in the solution of food security problem. It is argued that the tendency of demand for food has an increasing trend and the development of food markets still remains one of the most important challenges of contemporary world.

The study analyzes the markets of Georgian agrofood products and studies the modern challenges in these markets. Comparative analysis of Georgian and Spanish food markets is conducted.

The article estimates the positive and negative trends of export of Georgian agro-food products as well as the possibilities of diversification of products in the food markets.

According to "The Impact-Immediacy" matrix the hindering factors of Georgian agro products export are identified. Using the "Priority Scheme" the priorities of economic policy stimulating the export of agro-food products are revealed.

In terms of geographical orientation of the export the possibilities of diversification of food markets are discussed, especially in European markets. The conclusion is made that, in general, European food market is characterized by the growing tendency. However, in these markets there is no similar tendency for Georgian agro-food products.

According to the study of the share of Georgian products and export growth rates in different countries the positive correlation between these variables is determined. But this circumstance is not considered to be sufficient for the positive impact on the economic growth.

The conclusion is made that using effective mechanisms of export stimulation allows the diversification of export of Georgian agro products on the traditional but growing food markets on the one hand and on the other hand the expansion of geographical area of export markets. 